\title{
La pericia perceptivo-motriz y la cognición en el deporte: Del enfoque ecológico y dinámico a la enacción
}

\author{
Carlos Avilés ${ }^{1 *}$, Luis M. Ruiz-Pérez², José A. Navia², Natalia Rioja ${ }^{3}$ y David Sanz-Rivas ${ }^{4}$ \\ ${ }^{1}$ Facultad de Educación. Universidad Complutense de Madrid. \\ ${ }^{2}$ Facultad de Ciencias de la Actividad Física y del Deporte. Universidad Politécnica de Madrid \\ ${ }^{3}$ Facultad de Ciencias del Deporte. Universidad de Castilla-La Mancha \\ ${ }^{4}$ Facultad de Ciencias de la Salud. Universidad Camilo José Cela (Madrid)
}

\begin{abstract}
Resumen: En el presente artículo se discuten tres corrientes teóricas alternativas al cognitivismo, para explicar el desarrollo hacia la pericia en el ámbito del aprendizaje y del control motor en deporte, haciendo especial énfasis en el enactivismo. En la primera parte se tratan las principales nociones de la psicología ecológica, como la regulación del movimiento, la percepción directa o la reciprocidad entre percepción y acción. A continuación se explican las principales aportaciones de la teoría de los sistemas dinámicos a la comprensión de la coordinación del movimiento, de la emergencia de la motricidad y de la interacción de las diferentes variables o restricciones. En el siguiente apartado, se expone el enfoque enactivo como una extensión conceptual proveniente de las ciencias cognitivas y que trasciende a los otros paradigmas. Desde esta orientación, se aboga por una fusión entre el cuerpo y la mente del deportista que es indisociable al medio ambiente y que se opone al dualismo y reduccionismo imperante. Por último, se presentan algunas directrices y aplicaciones de investigaciones enactivas que en la actualidad están en pleno desarrollo.

Palabras clave: Enactivismo; cognición; acoplamiento informaciónmovimiento; post-cognitivismo; deporte; pericia.

Title: Perceptual-motor expertise and cognition in sport: From ecological and dynamic approach to enaction.

Abstract: In this paper three alternative theoretical streams to cognitivism, with particular emphasis on enactivism are discussed in order to explain the sport skill acquisition process and human control of movement. In the first part, main concepts of ecological psychology are outlined, like movement regulation, direct perception or mutual interdependence between perception and action. Afterwards, using dynamical model as background, some concepts of motor skill coordination are explained (i.e., constraints or movement emergency). The next section will be focused on enactive approach as a conceptual extension from cognitive science that transcends the other paradigms. From this orientation, it calls for a mind-body fusion of the athlete, which is inseparable to the environment and opposing the prevailing dualism and reductionism. Finally, some guidelines and applications enactive research that are currently being developed are presented.

Key words: Enactivism; cognition; information-movement coupling; postcognitivism; sport; expertise.
\end{abstract}

\section{Introducción}

El estudio de los deportistas expertos ha conocido un desarrollo importante durante las últimas décadas. La pericia en el campo del deporte ha sido estudiada principalmente desde una perspectiva cognitiva (Ruiz, Sánchez, Durán y Jiménez, 2006; Williams y Davids, 1998). La influencia del computacionalismo implicó el diseño de estudios en laboratorio muy lejanos al contexto habitual y exigente en el cual se desempeñaban los atletas. Los investigadores analizaban qué era lo que ocurría al interior de la mente y estudiaban cómo se llevaban a cabo una secuencia de mecanismos internos dentro de la caja negra de cada deportista. Importantes nociones cognitivas y prescriptivas han marcado muchos años de investigación: percepción indirecta, procesamiento de la información, representaciones, símbolos, programas motores, memorias, cálculos, etc. (Schmidt, 1991; Temprado, 1994). Sin embargo, con el paso del tiempo, la metáfora hombre-ordenador no ha parecido ser la más adecuada para representar el funcionamiento humano y el cognitivismo ha recibido numerosas críticas desde posiciones muy diferentes (Moe, 2005; Noë, 2010).

Este descontento y reticencia a aceptar la hegemonía cognitiva se ve reflejado explícitamente en el estudio de Abernethy y Sparrow publicado en 1992. Estos autores adoptaron una mirada kuhniana para explicar los procesos

* Dirección para correspondencia [Correspondence address]: Carlos Avilés Villarroel. C/ Rector Royo Villanova s/n. Ciudad Universitaria. C.P.: 28040. Madrid (España).

E-mail: caviles@pdi.ucm.es de crisis, de emergencia y de evolución de las teorías protagonistas del progreso científico dentro del campo del comportamiento motor, para llevar a cabo un análisis notable sobre el contenido y citación de los principales estudios publicados en el Journal of Motor Behavior. De esta forma, demostraron que los conceptos provenientes de las teorías ecológicas y dinámicas habían evolucionado de forma ostensible y que esto había contribuido al asentamiento de estos paradigmas en parte de la comunidad científica (Araújo, 2013).

Posteriormente al periodo de crisis, de controversia y de debate teórico, los estudiosos del deporte y de otras áreas del saber como la filosofía de la mente, neurociencias o las ciencias cognitivas, se han mostrado partidarios de crear vínculos entre paradigmas. La esperanza es la unificación teórica para explicar la gran variedad de fenómenos, tareas y habilidades implicadas en el ámbito del desarrollo, aprendizaje y control motor. Un gran número de investigadores se aproxima a una perspectiva híbrida entre el cognitivismo y la perspectiva ecológica-dinámica (Abernethy, 1998; Berthoz, 1997; Colley, 1989; Crognier y Féry, 2007; Keil, Holmes, Bennett, Davids y Smith, 2000; Nitsch y Munzert 2002; Norman, 2002; Paillard, 1991; Ripoll, 2009; Ruiz, 2003a; Ruiz, 2003b; Temprado y Laurent, 1995; Vickers, 2009; Williams, Davids y Williams, 1999).

Por otra parte, un grupo alternativo se ha decantado por un mestizaje de los postulados filosóficos, teóricos y metodológicos del enfoque ecológico y de los sistemas dinámicos (Araujo, Davids y Hristovsky, 2006; Beek, Jacobs, Daffertshofer y Huys, 2003; Davids, Button y Bennett, 2008; Davids, Handford y Williams, 1994; Davids, Williams, Button y Court, 2001; Handford, Davids, Bennett y Button, 1997; Sa- 
velsbergh, Van der Kamp, Oudejans y Scott, 2004; Temprado y Montagne, 2001; Warren, 2006b).

Pero estos enfoques no son los únicos en criticar al cognitivismo (García y Calvo, 2011). Complementariamente, la teoría de las contingencias sensorimotoras propuesta por O’Regan y Noë (2001) manifiesta que ver es una actividad exploratoria o una forma de actuar mediada por el conocimiento de las consecuencias de lo que pueda acontecer. Para Noë (2010) la consciencia no es un estado que sobreviene solo en nuestro interior. El significado no está dentro de la cabeza o exclusivamente en el cerebro, más bien es producto de lo que hacemos activamente durante una reciprocidad constante con el mundo que nos rodea.

Si bien existen diferentes posicionamientos teóricos que constituyen los enfoques corporeizados (Wilson, 2002). Las nuevas ciencias cognitivas se caracterizan por lo que Rowland (2010) y Pérez (2010) denominan las cuatro Es, es decir, Enactiva, (enacted), Embebida ${ }^{1}$ (embedded), encarnada (embodied) y extendida (extended). Lo que diferencia estos enfoques recientes de los anteriores es que sitúan la mente en el mundo, la saca de los límites del cráneo y la pone en relación con el resto de las partes del cuerpo y con el entorno.

El objetivo principal de este artículo es acercar a los especialistas de la Psicología del Deporte y de las Ciencias del Deporte en general, a las teorías no cognitivistas y no representacionistas que en la actualidad existen y que pueden explicar la evolución hacia el comportamiento experto y el funcionamiento de los deportistas.

Otra de las aspiraciones que se pretende en este documento, es proponer al enactivismo como un enfoque que puede llegar a beneficiar a las Ciencias del Deporte, por su clara oposición al dualismo cartesiano y por su enfoque global de la persona. Ésta perspectiva en sus diferentes vertientes más conservadoras o más radicales (Di Paolo, Rohde y De Jaegher, 2010; Thompson, 2011; Varela, Thompson y Rosch, 2005; Hutto, 2013), se interesa por la acción en situación del deportista, lo que abarca tanto a la cognición más sensorimotora como a la cognición más intelectual o superior. Sin duda, la mente y la cognición están en el centro del debate científico porque estos conceptos contienen todas las dimensiones humanas: perceptiva, emocional, motriz, intencional, reflexiva, etc. La radicalidad que adopta el científico enactivo se relaciona con su grado de acercamiento a las raíces esenciales del enactivismo y con su nivel de distanciamiento frente a los principios fundacionales del cognitivismo.

Cabe resaltar que cuando un deportista está actuando lo hace en su globalidad poniendo en acción todos los recursos que posee para resolver los problemas que su actividad deportiva le propone, es un sistema acoplado, y que desarrolla una historia de acoplamientos que le hace cada vez más eficaz en sus decisiones. Para Masciotra, Roth y Morel (2008),

\footnotetext{
${ }^{1}$ Basándose en Rowland (2010), Pérez en su artículo destaca como el término inglés embedded a pesar de que habitualmente se traduce como incrustado, él prefiere la traducción embebido que significa encajar, embutir, meter algo en una cosa, lo que le permite hablar de las 4 Es, de los nuevos enfoques postcognitivos.
}

la disponibilidad mental del deportista sería un tipo de consciencia activa presente en el aquí y en el ahora, "en una mente que se funde en la acción y que no da lugar a pensamientos diseminados" (p. 116).

La intencionalidad del experto, se relaciona con sus experiencias vividas adquiriendo una red de acciones potenciales que siempre estarán listas para ser activadas o disparadas en una especie de toma de decisiones creativa que no es meramente algo predeterminado y que se refleja en su corporalidad. Es decir, es una pericia en la que los puños tienen intenciones: "hay que pensar con las manos" (...) "el karateca sitúa su cognición en la relación entre su cuerpo y el mundo" (p. 130).

En consonancia con lo anterior, Noë (2010) destaca la importancia de los hábitos en el proceso de alcanzar la pericia. El experto no necesita reglas ni normativa ya que su alta sensibilidad permite que su destreza en responder sea activada por las circunstancias del contexto. Este autor se opone a una visión de la pericia como algo razonado e intelectual indicando que "no tenemos una relación de novatos y de extraños con el mundo" (...) "la pericia requiere la ausencia de cuidado y de deliberación que el intelectualista considera, de forma errónea, ser el rasgo distintivo de nuestra vida mental" (p. 131). Aludiendo a la globalidad de la cognición, merece la pena resaltar que el termino conocer implica un abanico de verbos tan variados como percibir, sentir, experimentar, entender, saber y razonar. El ser humano responde como un todo, como una unidad indivisible.

Aunque tienen diferencias particulares, los enfoques ecológico, de los sistemas dinámicos y el enactivo, poseen aspectos complementarios y son compatibles entre ellos. Frente al debate sobre la existencia de representaciones internas, estas perspectivas postulan su irrelevancia para explicar cómo emerge el comportamiento a través de acoplamientos entre el agente o deportista y su nicho ecológico. La evolución conductual del ser humano es producto de factores filogenéticos, ontogénicos y experienciales.

En este artículo se abordarán indirectamente contenidos relacionados con lo que es la cognición en su orientación más amplia incluyendo la percepción, la toma de decisiones, la regulación perceptiva del movimiento, la emergencia de la coordinación, el conocimiento enactivo y la adquisición de habilidades que forman parte del desarrollo de la pericia perceptivo-motriz y cognitiva. Para ello, se han establecido tres partes principales. En primer lugar, se expondrán los conceptos más relevantes de la perspectiva ecológica. En segundo lugar, se mencionarán brevemente las ideas fundamentales de la teoría de los sistemas dinámicos. Finalmente, se abordará el enfoque enactivo con un desarrollo de sus nociones más relevantes.

\section{El Enfoque ecológico y percepción directa}

El enfoque ecológico y la teoría de la percepción directa tienen su génesis principal en la obra de Gibson (1979/1986), en el replanteamiento sistemático de los postulados teóricos 
del enfoque cognitivo (e.g., Michaels y Carello, 1981) y en las aportaciones de los sucesores de James J. Gibson a partir de los años ochenta (e.g., Michaels y Beek, 1995).

En el ámbito de la investigación en Ciencias del Deporte o de la Psicología del Deporte, los postulados del enfoque ecológico se han aplicado para explicar cómo el ser humano percibe la información y cómo la detecta para controlar o regular sus movimientos. Se han estudiado diversos tipos de acciones, como por ejemplo la locomoción al subir escaleras (Warren, 1984) o la interceptación de una pelota en tenis de mesa (Bootsma y Van Wieringen, 1990). Como se verá, para el estudio de la percepción y del control perceptivo-motor es fundamental comprender la naturaleza o estatus de la información y su vínculo o dependencia que tiene con el movimiento del deportista.

El deportista experto está constantemente sometido a una alta exigencia espacial y temporal, dispone de breves periodos de tiempo para ver y aprehender la trayectoria del móvil, teniendo que superar las posibilidades de su sistema visuomotor, y sin embargo, es capaz de responder de forma rápida, inmediata y con una gran precisión y eficacia. Como la información es de gran riqueza, puede ser percibida directamente. El enfoque gibsoniano se decanta hacia la posibilidad de que el deportista pueda regular continuamente su movimiento sobre la base de la información disponible de manera directa en el medio, para estar ubicado en el lugar oportuno y en el momento preciso.

En deportes como el Tenis, Squash o Bádminton, se pueden observar múltiples situaciones rápidas e imprevisibles donde parece difícil planificar con anterioridad el control de todas las articulaciones, músculos y movimientos que se ponen en juego para conseguir acciones eficaces. En este tipo de situaciones dinámicas y de urgencia, el acceso a representaciones internas y el empleo de computaciones necesitaría tiempos relativamente largos de procesamiento de la información visual, que impedirían que se pudiese responder a las demandas de la pelota o del volante. Pensemos que los grandes jugadores asiáticos pueden llegar a golpear el volante a más de $300 \mathrm{~km} / \mathrm{h}$. Como consecuencia, uno de los problemas principales que plantea el enfoque cognitivo es el coste funcional que esto supondría (Benguigui, 1997).

Del mismo modo, y siguiendo con una mirada cognitiva, el jugador de la NBA tendría dificultades para adaptar el gesto a los cambios continuos y rápidos de la trayectoria del balón que emergen de la dinámica del juego. Pareciera que la utilización de estructuras mentales prescriptivas no es imprescindible porque la información que especifica la acción está directamente disponible en la escena visual. Como indica Wheeler (2001) sería un comportamiento inteligente online que favorecería la producción de una respuesta fluida y adaptable a los estímulos sensoriales concurrentes.

En la gran mayoría de las situaciones, las acciones son llevadas a cabo mediante respuestas adecuadas, ya que el deportista es capaz de percibir directamente las affordances o posibilidades de acción que le brinda el medio ambiente en cada situación (Gibson, 1979/1986, p.127). Los enfoques cog- nitivos de percepción indirecta difícilmente pueden explicar estos fenómenos fluctuantes, inesperados y veloces mediante un proceso de enriquecimiento de índices o de esbozos tal como lo planteaba Marr en su texto publicado en 1985. Desde el punto de vista gibsoniano, la realidad visual no se construye, sino que es percibida o detectada directamente por el deportista.

No solo en el deporte tenemos ejemplos de una gran destreza, en la vida cotidiana se pueden encontrar casos interesantes que muestran una pericia perceptivo-motriz muy evolucionada en el ser humano. Por ejemplo, como conductores somos capaces de tomar una decisión instantánea y correcta al esquivar un coche que es una amenaza, en tan sólo una fracción de segundo. ¿Cómo podemos ser capaces de captar, codificar y descodificar la información para percibir el vehículo, de seleccionar y de recuperar un programa motor almacenado en memoria acorde a la necesidad surgida, y finalmente de programar los parámetros exactos de la respuesta para evitar la colisión en una ventana temporal tan limitada?

Se podrían encontrar infinidad de ocasiones en los que aflora la interrogante de cómo el ser humano se acopla a las situaciones y de forma rápida y precisa solventa los problemas que encuentra. Un portero de fútbol que debe parar un balón chutado a una velocidad intratable, un bateador en béisbol, un jugador de pádel, unos niños que en el recreo juegan a no ser golpeados por el balón que lanza con todas sus ganas su oponente o un bombero que debe actuar intuitivamente para salvar su vida durante un incendio.

En definitiva, nos encontramos ante el reto de explicar cómo se pueden llevar a cabo estas decisiones sin necesidad de tener que emplear procedimientos lentos y mentalmente costosos que impedirían su realización, y ello pone en discusión la forma en que los deportistas y los seres humanos perciben, deciden y actúan.

\section{El acoplamiento entre la información y el movi- miento}

Para Bardy (2006) la posibilidad de controlar la acción a través de un mecanismo de anticipación perceptiva, que utiliza la copia de órdenes motoras ya conocidas, es un proceso poco admisible. Esta copia de eferencia, funciona para movimientos simples donde la relación entre los diferentes niveles de organización es sencilla. Sin embargo, para los movimientos complejos como los deportivos, la discrepancia entre los mandos motores y las consecuencias perceptivas del movimiento es muy importante. Los enfoques postcognitivos como el ecológico explican esta regulación perceptivo-motriz de manera más simple sin tener que recurrir a operaciones mentales complicadas.

En relación con lo expuesto en las líneas precedentes, Bootsma (1993) señala que "los sistemas biológicos que han sufrido y sobrevivido a las fuertes presiones evolucionistas son bastante eficaces en la gran mayoría de las situaciones" (p. 14). Destacando la importancia de la relación circular en- 
tre la información y la acción Michaels y Beek (1995) han señalado que "el ciclo percepción-acción es visto matemáticamente como un conjunto de intercambios recíprocos que trazan los descriptores cinéticos (fuerza) sobre los descriptores cinemáticos (información)" (p. 261).

El flujo es el cambio de la configuración óptica que es producido por las fuerzas externas del contexto y por las fuerzas producidas por el propio deportista. El experimento inédito de Carlton, Carlton y Kim (1991), comentado por el propio Carlton en su revisión de 1992, muestra claramente la noción de acoplamiento perceptivo-motor y que recuerda la idea de ciclo funcional de Merleau-Ponty (1945/1985). Los investigadores modificaron algunas zonas de la superficie de una pista de tenis, logrando una desviación de la trayectoria de la pelota tras el bote. La corrección o ajuste involuntario del gesto del jugador, consistía en aumentar o disminuir la aceleración de la raqueta según la situación. Lógicamente, el bote rápido produjo un incremento en la aceleración de la raqueta y el bote lento provocó una reducción. Como resultado, se obtuvieron retrasos visomotores más cortos o más largos que fueron atribuidos al tipo de modificación de la información de cada condición.

Las leyes de control son mecanismos de regulación basados en la puesta en relación de una propiedad del flujo óptico y un parámetro del movimiento. Los cambios, las modulaciones de fuerzas que producen los deportistas dependen de las variaciones del flujo. Existe una causalidad circular porque las fuerzas que produce el atleta (fuerzas internas) son a la vez la causa y la consecuencia del flujo de información que estimula sus receptores (Bardy, 2006). De ésta manera, el acoplamiento percepción-acción sugiere la idea de que el movimiento crea la información y viceversa, la información crea el movimiento.

El control perceptivo-motor automático está estrechamente ligado a la visión periférica y a la visión para la acción. Para Milner y Goodale (1995) el sistema dorsal, permite regular y modular en continuo los segmentos corporales con la información actual en contextos variables y dinámicos. Posee una importante función en las habilidades de intercepción que requieren una regulación del efector. Por ejemplo, para coordinar la acción del pie sobre la tabla de batida en salto de longitud o para ajustar la acción de bateo en béisbol. En tenis ya existe evidencia de que los restadores aumentan sus porcentajes de predicciones correctas cuando responden en movimiento con su patrón de ejecución habitual en comparación con los pronósticos sólo perceptivos realizados en ausencia de movimiento (Farrow y Abernethy, 2003).

\section{La adquisición de la pericia perceptivo-motriz}

El enfoque ecológico explica el aprendizaje como un proceso de descubrimiento y optimización del acoplamiento información-movimiento lo que conlleva a una estabilización de las leyes naturales de control (Bardy, Delignières, Lagarde, Mottet y Zelic, 2010). Cuando el deportista sintoniza con su entorno aumenta su capacidad para detectar la informa- ción útil para actuar, capta las tonalidades funcionales de los objetos y situaciones. Éste progreso de su pericia significaría una mejora en la percepción de las affordances incrementando la eficacia en la utilización de la información.

Un comportamiento adaptativo sigue un criterio de eficiencia. Bootsma (1994) adoptando el lenguaje de Gibson menciona que "se calificaría de inteligente el comportamiento de un sistema que al percibir y actuar, minimiza igualmente el cálculo y las inferencias explotando de manera oportuna toda la información disponible en la situación” (p. 50). Para alcanzar dicho nivel, los investigadores neogibsonianos han señalado que el aprendizaje se produce principalmente por tres vías: la educación de la intención, educación de la atención y calibración del movimiento (Jacobs y Michaels, 2007; cf. Ibañez-Gigón, Travieso y Jacobs, 2011; Withagen y Chemero, 2009).

La educación de la intención consistiría en aprender a seleccionar aquellas utilidades-affordances emergentes del entorno sobre las que guiar la conducta que potencialmente permitirían alcanzar el objetivo deseado. Por ejemplo, para un delantero en fútbol, aprender a considerar un balón como chutable o pasable en función de la situación de juego y según el objetivo final que puede ser superar al contrario o marcar gol.

La educación de la atención supondría aprender a regular las acciones basándose en variables específicas o especificadoras del entorno. Esto supondría cambiar la atención de aquellas variables que, aunque pueden guiar la conducta, se relacionan de una forma baja o moderada (variables no especificadoras del entorno) hacia aquellas que lo hacen de una forma alta o completa (i.e., invariantes) con el entorno. Un ejemplo entre las variables que especifican plenamente las propiedades físicas del entorno más testadas experimentalmente ha sido la variable óptica $\tan (\tau)$ en sus diversas modalidades. Ella permitiría que el sujeto pueda percibir directamente el tiempo restante antes de la intercepción o de la acción (Lee y Reddish, 1981).

Por último, la calibración supondría la mejora del acoplamiento entre la información y la acción, en términos espaciales y temporales, y siempre en función de las capacidades morfológicas (i.e., tamaño) y funcionales (i.e., rapidez) del propio deportista. Esta calibración cambia con el entrenamiento permitiendo al atleta experto un mejor control y precisión. Por ejemplo, Bootsma, Houbiers, Whiting y Van Wieringen (1991) estudiaron la evolución de jugadores noveles de tenis de mesa en el aprendizaje del golpe de derecha. Tras 1600 ensayos de entrenamiento, los investigadores observaron una disminución de la variabilidad al momento del impacto. En efecto, la dirección del vuelo de la raqueta era mucho más consistente en el instante de la intercepción. De este modo, el aprendizaje se plasmó en una adaptación específica o calibración del acoplamiento percepción-acción permitiendo una regulación on-line de la acción. 
Adaptabilidad y variabilidad funcional del movimiento

Los movimientos del experto muestran respuestas consistentes sobre una serie de varios ensayos. Recientemente Bardy et al. (2010) mencionan algunas características importantes para analizar lo que identifica estos movimientos con relación a la variabilidad. Es decir, las desviaciones típicas son estables y reproducibles.

Aunque es necesario resaltar que los movimientos expertos nunca son ejecutados exactamente de la misma manera de una ejecución a otra. Es por ésta causa que los deportistas a menudo, adaptan la acción en curso realizando pequeños ajustes lo que implica un comportamiento de naturaleza funcional y no estereotipado. La adaptabilidad que manifiestan los deportistas expertos se relaciona con lo que es el control prospectivo que fue revisado por Montagne en 2005. Este mecanismo permite al deportista la producción de movimientos regulados con la información actual disponible en el entorno. De esta forma, la anticipación deja de ser predictiva (modelo cognitivo) para ser prospectiva, es decir, se redefine la anticipación como la capacidad para usar la información actual presente, o información acerca del futuro actual, ajustada a las máximas posibilidades funcionales de actuación del individuo (cf. Montagne, Bastin y Jacobs, 2008).

En uno de los estudios más notables sobre el timing del golpe de derecha en tenis de mesa realizado con jugadores expertos, Bootsma y Van Wieringen (1990) indicaron: "que la acción sea ejecutada más rápida o lentamente, lanzada un poco mas tarde o un poco más temprano, carece de importancia, siempre y cuando se mantenga un acoplamiento entre las variables perceptivas y de movimiento" (p. 28). En éste experimento, se descubrió una variabilidad compensatoria entre los componentes perceptivos y motores. Cuando el jugador empezaba a mover su brazo un poco más tarde, aumentaba la fuerza para reducir el tiempo de movimiento y de esta manera alcanzaba la pelota en su adecuado punto de golpeo. En otra tarea de intercepción durante el salto preparatorio (split-step) en el resto de servicio, los jugadores de tenis expertos mostraron un comportamiento global mucho más estable que los jugadores de menor nivel. Éste efecto fue encontrado en el momento del salto, de la fase de vuelo y de la caída. Probablemente, los restadores expertos detectaban en continuo la información, para adaptar la acción de sus pies a las variaciones de altura que el sacador efectuaba durante la fase del lanzamiento de la pelota (Avilés, Ruiz y Benguigui, 2006).

\section{Sistemas dinámicos y emergencia de la coor- dinación motriz}

En el estudio de la motricidad, del control y del aprendizaje motor aplicado al deporte, este enfoque de los sistemas dinámicos se ha aplicado principalmente en el análisis de la coordinación de los movimientos, objetando explícitamente la noción cognitivista de programa motor y considerando al deportista como un sistema que se autoorganiza relacionándose con el ambiente, y examinando cómo las constricciones afectan la estabilidad produciendo cambios hacía nuevos estados preferenciales (Torrents y Balagué, 2007).

De este modo, los partidarios de la teoría de los sistemas dinámicos tienen como propósito analizar cómo son las relaciones dinámicas que el deportista establece con su entorno, la evolución de las coordinaciones en el espacio perceptivo-motor de trabajo y los momentos de transición de la coordinación al control, y de éste a la pericia. También, los investigadores dinamicistas tienen como finalidad estudiar el papel que cumplen la exploración y el descubrimiento en el proceso de desarrollo de la pericia (Araujo et al., 2006; Beek et al., 2003; Davids et al., 2008).

La complejidad de la motricidad humana radica en el hecho de que el cuerpo está constituido por alrededor de $10^{3}$ músculos y $10^{2}$ articulaciones, que interactúan entre sí con una gran cantidad de receptores, que propagan durante la acción un número muy importante de señales hacia las células nerviosas (Bardy, 2006, p. 135). Entonces, si la interacción de tantos elementos entran en juego para cualquier movimiento. ¿Cómo surge la coordinación en una acción deportiva? Por ejemplo, atrapar una pelota que se aproxima es una habilidad bastante compleja ya que implica coordinar numerosos componentes del sistema neuro-músculoesquelético. Cada uno de estos elementos posee un cierto grado de libertad de funcionamiento. Pero, cuando analizamos la relación de todos estos componentes en los movimientos hábiles, el número de grados de libertad a controlar deviene enorme. Para solventar este problema Nicolai A. Bernstein propuso como solución sinérgica o la unión y relación de los elementos de la acción, para reducir el número de grados de libertad a controlar del sistema. El sistema motor y sensorial se ensamblan para producir una actuación adaptativa (Whiting, 1984).

Los conceptos de auto-organización y emergencia constituyen uno de los núcleos principales de la teoría de los sistemas dinámicos. Desde esta perspectiva, un nuevo patrón de comportamiento emana de la interacción de las diversas restricciones, constricciones, variables limitadorascanalizadoras del sistema o constreñimientos (constraints) (Ruiz, 2003b). En sus inicios, el estudio de la coordinación se llevó a cabo en tareas bimanuales y rítmicas que realizaba un sujeto. Actualmente, este paradigma ya se ha ampliado a programas de investigación donde se analiza el comportamiento motor o el desplazamiento espontáneo de deportistas en el mismo terreno de juego. En este marco, se puede citar como ejemplo el estudio de Palut, Zanone y Gurdjos (2004) en tenis. Estos investigadores han demostrado cómo la coordinación de ambos jugadores emergía de un acoplamiento visual entre ellos y de una consigna de juego específica que consistía en fatigar al contrario antes de marcar el punto.

Es importante destacar la crítica que realizaron Davids et al. (2008) frente a la posibilidad de que en el SNC y más pre- 
cisamente en el cerebro, resida un homúnculo responsable de los programas motores y de las acciones del deportista. La analogía de un titiritero controlando cada movimiento de una marioneta ejemplifica la reprobación hacia el enfoque cognitivo que se fundamenta en un mecanismo o sistema de control central y jerárquico. La teoría de los sistemas dinámicos "es un enfoque que considera al aprendiz como un sistema neurobiológico complejo. Éste, se compone de grados de libertad o subsistemas que son independientes, pero que a su vez interactúan entre ellos” (p. 224).

Un sistema complejo tiene la particularidad que puede adoptar comportamientos diferentes por medio de relaciones no lineales entre sus propios componentes y las variaciones del medio ambiente. Por ejemplo, a nivel cerebral, la actividad dinámica del ser humano no responde a relaciones determinadas de causa y efecto, entre la activación de un área localizada y el comportamiento resultante. Si el cerebro esta compuesto por millones de neuronas y sinapsis es fácil advertir la complejidad de su funcionamiento (Oullier, Lagarde, Jantzen y Kelso, 2006). El cerebro opera de forma distribuida a partir de interconexiones masivas y no con reglas secuenciales. En relación con la emergencia neuronal Varela et al. (2005) indicaron que "una neurona participa en muchos modelos globales como el descrito y tiene escasa significación cuando se la toma aisladamente” (p. 123).

\section{Pericia perceptivo-motriz}

Newell (1986) propuso una perspectiva teórica interesante para explicar la emergencia de la coordinación mediante la interacción de restricciones o variables ligadas al organismo, al medio ambiente y a la tarea. Las relacionadas con las características del propio deportista como su cognición, intención, motivación, emoción, nivel de pericia, factores genéticos, atención, ansiedad, memorias, morfología, etc. Las vinculadas con el medio ambiente o contexto de práctica como las variables informacionales, fuerza de gravedad, condiciones ambientales, luz o temperatura. Por último, las relacionadas con las características de la tarea a acometer que implican los objetivos, las instrucciones, el modelado, las reglas de actuación, los materiales o instrumentos de cada deporte, las tácticas empleadas, etc.

El deportista que aprende no comienza de la nada, siempre posee requisitos, condiciones, recursos propios y estados preferenciales para enfrentarse a un nuevo cometido o desafío. A medida que se evoluciona hacia la pericia, el deportista aprende a utilizar de manera óptima la coalición de las diferentes constricciones. Apoyándose en las ideas Bernsteinianas, Newell (1985) expone tres etapas de desarrollo de la pericia. La primera que se relaciona con el ensamblaje de un nuevo patrón de coordinación. La segunda que hace referencia a la ganancia de control y de adaptabilidad de la estructura coordinativa. En la tercera, el deportista aumenta su habilidad y eficiencia reduciendo el coste mecánico y metabólico, explotando las posibilidades que el sistema percepti- vo-motor le ofrece para solucionar los problemas a los que se enfrenta.

Los profesores y entrenadores deberían utilizar una pedagogía no lineal (Chow, Davids, Hritoski, Araújo y Passos, 2010; Davids et al., 2008) entendida como la consideración de los constreñimientos dentro del proceso de aprendizaje para favorecer el desarrollo de la competencia de los individuos, convirtiéndose los profesores, entrenadores o psicólogos en facilitadores de las actividades de exploración en la búsqueda de las soluciones más apropiadas al problema planteado, considerando que no siempre más supone mejor. En este sentido, es importante comprender que no existe un patrón técnico idealizado y que los entrenadores en las prácticas deben fomentar la eficacia más que la forma del gesto exclusivamente. Por ejemplo en el tenis profesional, hay muchos jugadores capaces de sacar por sobre los $200 \mathrm{~km} / \mathrm{h}$ con estilos técnicos muy diferentes. El modo preferencial descubierto y elegido por éstos sacadores es producto de miles de repeticiones, de aciertos, de errores y de la interacción de restricciones comentadas anteriormente.

En cuanto a las condiciones que favorecen el aprendizaje, Temprado y Montagne en 2001, mencionan que el efecto de las variables clásicas utilizadas desde una perspectiva cognitiva para estudiar los movimientos simples, también deben ser analizadas para observar la adquisición y el desarrollo de las coordinaciones complejas como las encontradas en las situaciones deportivas. De esta manera, una gran cantidad de variables podrían ser utilizadas por el entrenador. Se podría manipular la frecuencia del conocimiento de resultados, el feed-back o la variabilidad de las condiciones de práctica para movimientos complejos.

El profesor o entrenador podría utilizar o modificar los materiales específicos del deporte o adaptar el terreno de juego. También se podría guiar la atención del deportista hacia aspectos de su postura o hacia los efectos de su propio movimiento, pero al mismo tiempo no dar consignas u órdenes que limiten la búsqueda de soluciones relacionadas con la energía, la mecánica y la atención utilizadas. En definitiva, como lo exponen Moreno y Ordoño (2009), la modificación de las variables es lo que estimulará la capacidad adaptativa del deportista hacia una nueva dinámica de movimiento.

Para concluir este apartado, es muy interesante destacar la inclusión de variables cognitivas en estudios de carácter dinámico (Summers, 2004), ya que como indica Temprado y Montagne (2001) la cognición no está excluida del todo dentro del lenguaje dinamicista:

Podemos sorprendernos al constatar que las variables "cognitivas" puedan encontrar su lugar dentro del enfoque dinámico donde las representaciones y los modelos internos no forman parte de la conceptualización. Esto no es contradictorio si consideramos que todas las informaciones que son dadas a los sujetos actúan como restricciones que permiten explorar de mejor manera el espacio perceptivo-motor de la tarea (p. 151). 


\section{Enacción y deporte}

Del mismo modo que el enfoque ecológico, el enactivismo le otorga un rol fundamental a la acción o al movimiento para la percepción. El punto de vista enactivo atribuye también gran importancia a la auto-organización y emergencia que son conceptos claves de la teoría de los sistemas dinámicos (De Jaegher y Di Paolo, 2007). Un análisis crítico sobre las diferencias y afinidades de estas tres aproximaciones está presente en el texto de Varela et al. (2005). El enactivismo, como expone Rowland (2010) es uno de los enfoques postcognitivos que tratan de explicar las estrechas relaciones del sujeto con su entorno, y cómo estos vínculos son el origen de su cognición.

El enactivismo es producto principalmente de la evolución de las ciencias cognitivas y en cierta forma es un enfoque que abarca a los demás. Por este motivo, no es de extrañar el hecho de que algunos científicos del deporte con intereses o posicionamientos teóricos diferentes, ya lo hayan incorporado en sus programas de investigación (cf. Bardy, 2011; Laurent y Ripoll, 2009). En realidad es una convergencia de ideas que a día de hoy, han constituido un programa científico en crecimiento y que va más allá de las críticas a la teoría de procesamiento de la información. Ésta perspectiva se aproxima al naturalismo, a la fenomenología y se distancia del funcionalismo, dualismo y reduccionismo (Di Paolo et al., 2010).

Desde una perspectiva evolutiva, ya desde los primeros días de vida, el ser humano se caracteriza por un comportamiento autónomo e inteligente a pesar de no disponer de un conocimiento abstracto desarrollado. Para Varela (2000), surge de la motricidad humana una flexibilidad extraordinaria que es observable cuando el bebé experto interactúa con los objetos que constituyen su entorno perceptivo-motor.

A lo largo de los años, el ser humano irá adquiriendo el conocimiento que es el producto de adaptaciones situacionales por medio de interacciones no lineales entre los elementos del sistema y el ambiente. Desde una orientación enactiva, las habilidades cognitivas básicas (e.g., percepción, movimiento y aprendizaje) son la base para la emergencia de las performances cognitivas superiores como la imaginación, la comunicación, el lenguaje y el razonamiento. En palabras de Varela: "El gran invento de la vida es la unión captorneurona-músculo. A partir de ahí, hay una complejidad creciente de la autoorganización cerebral" (p. 48).

El concepto de enacción fue utilizado en los años sesenta por Jerome Bruner cuando hacía referencia al rol fundamental de la representación y el conocimiento enactivo en el desarrollo cognitivo del ser humano durante el estadio sensoriomotor: "una etapa en que la acción y la experiencia externa están fundidas. Piaget se refiere a esta primera parte de la inteligencia sensoriomotora, como aquella en que las cosas son vividas más que pensadas" (Bruner, 1966/1980, p. 38).

Si para Bruner el conocimiento enactivo era el que se adquiría y manifestaba a través de la acción. También habría que considerar los trabajos de Poincairé (1907) sobre el pa- pel del movimiento en la construcción de la percepción espacial o las afinidades filosóficas con la fenomenología existencial de Merleau-Ponty (1945/1985), la tradición de la psicología de los países del Este sobre el papel de la acción en la conciencia o el pensamiento educativo de Dewey (1929). En la actualidad desde campos muy diversos como la inteligencia artificial, la neurobiología o la biología evolutiva se destaca el papel de las acciones, y de la cognición como una cognición encarnada y situada.

Las líneas precedentes se pueden comprender a través de la siguiente experiencia: si lanzamos burbujas de jabón al aire, una niña pequeña de año y medio interceptará algunas de ellas sin mayores inconvenientes. En su corta vida nadie le habrá enseñado a hacerlo ni tampoco tendrá incorporado en su base de conocimientos el cómo hacerlo, simplemente el comportamiento emergerá, vivirá la experiencia y aprenderá de ella. Con la práctica en situaciones similares su habilidad interceptiva se perfeccionará hacia un nivel mayor de pericia.

Un deportista es un agente en movimiento, no un pensador inmóvil y, por consecuencia, sus manifestaciones deberían ser estudiadas durante la temporalidad presente de su accionar. La tarea principal del deportista no es el descodificar o analizar en profundidad lo que ocurre, sino más bien, responder óptimamente o rápidamente a los cambios que se producen en el campo de juego. La gran capacidad adaptativa que caracteriza a un deportista excelente es producto de un largo recorrido evolutivo y de su formación deportiva que implica procesos de aprendizaje de habilidades de diversa naturaleza.

Las intuiciones e ideas precursoras a la enacción se fueron gestando y madurando en la dinámica de colaboración entre Maturana y Varela durante los años setenta y ochenta (Maturana y Varela, 1996, 2004). El enfoque enactivo fue propuesto inicialmente por Francisco Varela hace poco más de veinte años (véase Varela, 1996). Posteriormente Varela y sus colaboradores sentaron las bases fundacionales para el desarrollo de un programa de investigación en torno a la enacción (Varela et al., 2005). Para estos autores cognición es enacción que es un neologismo que significa enactuar o "hacer emerger el sentido a partir de un trasfondo de comprensión" (p. 176). Por tanto, el conocimiento es producto de esta capacidad de entendimiento que "está arraigada en la estructura de nuestra corporización biológica” (p. 177).

$\mathrm{El}$ argumento fundamental es que a lo largo de la filogénesis y de la ontogénesis, el sistema nervioso ha integrado e incorporará relaciones, o conexiones, entre el movimiento, la percepción y el medio ambiente. Estos acoplamientos han permitido la supervivencia de la especie humana y por tanto son el producto de la evolución biológica, de la selección natural y de las experiencias vividas por cada individuo a lo largo de su historia de vida. Para estos autores conocer consistiría en capturar el sentido común, o conocimiento práctico, que surge y se renueva durante las experiencias mismas. En este sentido se desarrollarían fuertes ensamblajes o acoplamientos percepción-acción-cognición (enacción) indisociables con el entorno en el cual evoluciona el organismo. 
Di Paolo et al. (2010) mencionan que en esta nueva era postcognitiva, el enactivismo le da un papel central al cuerpo ya que éste último se encuentra en la génesis de la mente y la cognición. La enacción engloba las facultades cognitivas más elementales como la percepción visual a las más abstractas como el pensamiento o la reflexión e incluye a la emoción que recupera su valía como fenómeno de estudio. En otras palabras, las ideas enactivas suponen un cambio metodológico trascendental porque abordan lo que es el conocer, el entender y la experiencia como una actividad formativa que descubre significados a través de la acción. En éste enfoque se entrelazan conjuntamente cinco nociones fundamentales que le aportan identidad y lo constituyen: autonomía, dar sentido (sense-making), emergencia, corporización o encarnación (embodiment) y experiencia.

El vínculo entre autonomía y sense-making es sin duda una de las tantas aportaciones sobresalientes de Varela (1984). Para Thompson (2011), una de las características esenciales de los seres vivos es que viven en condiciones de inestabilidad y precariedad. Esto requiere de un constante proceso de adaptabilidad y de dar sentido a las cosas lo que se relaciona con el concepto de intencionalidad. Frente a los cambios ambientales los seres vivos adoptan una sensibilidad o actitud receptiva y un significado de valores y normas que son fundamentales para dirigir u orientar sus respuestas hacia ellos.

En cuanto a las condiciones de normatividad que deben ser satisfechas o cumplidas por el sistema, Barandiaran, Di Paolo y Rohde (2009) aportan ejemplos muy esclarecedores. Los movimientos en forma de temblores o espasmos de un enfermo de Parkinson, no son una respuesta a las normas u objetivos. Por el contrario, un sistema compuesto por un gatito que es calentado por su madre, si que satisface la norma consitente en mantener la temperatura dentro de los límites de viabilidad. En definitiva, "las normas son relacionadas directa o indirectamente con las necesidades vitales (automantenimiento de la infraestructura biológica del agente) o bien son adquiridas y corporizadas en otras formas de vida autosostenida (psicológica, cultural, etc.)" (p. 372).

Un llamamiento hacia un enactivismo radical es el que proclama Hutto (2013) oponiéndose a cualquier tipo de contenido que implique la utilización de representaciones internas y abogando por un saber cómo o sentido común que es el producto de la acumulación de experiencia y de la formación de hábitos, disposiciones o modos de actuación. Esta propuesta se relaciona con la idea de construcción de una normatividad que es fundamental para un principiante en proceso de ser experto en cualquier habilidad. La repetición y la práctica son los cimientos de un conocimiento práctico no proposicional que implica una ausencia de deliberación y que es de gran importancia en el éxito de las acciones deportivas (Noë, 2010).

El defensor experto en voleibol manifiesta regularidades comportamentales, normas o tendencias para impedir la perdida del punto cuando el balón toca el suelo. El estudio de sus apreciaciones y preferencias permite acceder a su cognición (Récopé, 2006; Récopé, Fache y Rix, 2008).

Para Di Paolo et al. (2010) el término sense-making es "creación y apreciación de significado" (p. 39). Este concepto fundamental implica una actividad que es activa por parte del agente, es decir, el sentido o significado debe ser generado mediante interacciones transformacionales. En este punto hay una diferencia con el enfoque ecológico de Gibson ya que el enactivismo implica "una actividad que deja una huella formativa y que no es simplemente una extracción de ingenua información como si ésta estaba ya presente..." (pp. 3940). Según Varela et al. (2005) Gibson crea su teoría de la percepción otorgándole exclusividad al medio ambiente indicando que "ese intento pasa por alto no sólo la unidad estructural (autonomía) del animal sino la codeterminación de animal y ambiente..." (p. 238).

Recientemente, los partidarios de la enacción se han interesado también en la dimensión social de la cognición, analizando cómo emerge el significado mediante las interacciones de las dinámicas colectivas entre dos o varios agentes. Es así como De Jaegher y Di Paolo (2007) destacan la noción de creación de sentido cooperativo o participativo (participatory sense-making) que corresponde a la evolución de un dar sentido individual hacia un dar sentido colectivo. Los investigadores analizan las dinámicas de los patrones de coordinación entre dos o más sistemas o agentes y sus momentos de ruptura. También se estudia cómo se produce la interacción social y la capacidad para compartir significados y entender a los demás. Complementando lo descrito anteriormente, Di Paolo et al. (2010) exponen diversas situaciones en las cuales se puede analizar la cognición social. La generación de valores o incluso cuando los agentes no están presentes físicamente y deben crear el sentido a través de correos electrónicos. Sin duda el estudio de lo social tiene una gran relevancia en deporte. Por ejemplo para conocer las intenciones de los adversarios o para coordinar las acciones de defensa y ataque con los compañeros de equipo en deportes colectivos.

\section{Hacia un nuevo concepto de representación}

El enactivismo no acepta el dualismo de un mundo que tiene rasgos pre-establecidos y fijos donde el organismo se adapta a él mediante representaciones. La enacción se plantea explícitamente como una vía media entre el dualismo y el monismo. No admite la separación entre el organismo y el mundo externo como sucede con la teoría de procesamiento de la información. Como en la teoría de los sistemas dinámicos, el concepto de emergencia es fundamental desde el punto de vista enactivo. La enacción sostiene que el organismo y el ambiente se codeterminan mediante la conexión de múltiples niveles de sub-redes sensorio-motrices para hacer emerger un mundo de significación. El medio ambiente es enactuado, se conforma continuamente y por lo tanto la percepción de él es enactuación sensorio-motriz (Varela et al., 2005). 
El rol que juega la representación y la memoria estaría externalizado en el mundo mediante la acción. Es en este punto en el que aparece el debate sobre la representación de las acciones que de forma clara ha sido defendida por los enfoques cognitivos (Tenenbaum y Land, 2009) y rechazado por autores como Dreyfus y Dreyfus (1986) cuando de expertos se trata. Para éstos últimos, los expertos no necesitan representaciones mentales para llevar a cabo las habilidades que dominan (Peña, 2010), sus respuestas se basan en la intuición (Ruiz, 2012). Wheeler (2001) considera que el ser humano, en nuestro caso el deportista, codifica el mundo en términos de posibilidades de acción, y de existir representaciones, éstas estarían orientadas a la acción (AORs- Activity Oriented Representations). Estas representaciones serían el conocimiento sobre cómo actuar, siendo de carácter egocéntricas, específicas y dependientes del contexto de actuación. Con ello lo que se indica es que la propia representación va más allá del cráneo de los deportistas y se extiende al medio en el que actúa.

Desde el punto de vista enactivo la representación es definida como una actividad, un proceso o una acción que consiste en volver hacia o que refleja el presente (Havelange, Lenay y Stewart, 2003). Por consiguiente, ella ya no sería vista como un estado mental con un contenido que precede a la realidad. El sujeto y el objeto se co-determinarían o coocurrirían en el tiempo presente de una relación recíproca. Se ilustra éste acoplamiento con el ejemplo de la acción de percibir los olores y los colores que es diferente y particular en cada situación porque cada sujeto debe oler y ver objetos que ya no están preestablecidos de forma previa sino que hay que descubrirlos. La acción (cognición enactiva) tiene efectos directos sobre la sensación formando parte esencial de la percepción.

\section{La senda de aprendizaje hacia la pericia y conoci- miento enactivo}

Ya ha quedado patente la estrecha relación de la enacción con el aprendizaje y su producto en forma de conocimiento. En las primeras fases durante la adquisición de cualquier habilidad, el aprendiz posee intenciones, sin embargo, la ejecución de sus movimientos no será óptima. Mediante la praxis, la relación entre su intencionalidad y la acción motriz se acoplará de mejor forma. De manera que la unidad mente-cuerpo reflejará su máxima expresión en la maestría con la que un deportista experto funciona ya que "con la práctica, la conexión entre intención y acto se intensifica, hasta que al final la sensación de diferencia entre ambos desaparece casi por completo" (Varela et al., 2005, p. 54).

Desde el punto de vista enactivo de la percepción, éste no es un proceso pasivo que ocurre exclusivamente en el cerebro. Para Noë (2004) "la percepción es una forma de actuar" (...) "es algo que hacemos" (p. 1), "es una especie de actividad corporal habilidosa" (p. 2). La visión adquiere un sentido cinestésico similar al que ocurre cuando se palpa o se manipula un objeto. El contenido o consciencia de algo no se obtiene totalmente de una sola vez. Se necesita descubrir y explorar con el movimiento de los ojos y del cuerpo para percibir o comprender de forma implícita el contenido de la escena visual. Esto implica conocer tácitamente las consecuencias del movimiento sobre la estimulación.

El conocimiento enactivo expresa lo que Pasquinelli (2007) denomina un aprendizaje por la acción. Todo esto se manifiesta en la precisión del movimiento del pincel sobre el lienzo, de los ajustes precisos que realiza un piloto de formula uno sobre el volante o de la extraordinaria simpleza con la que un tripulante hace aterrizar su avión sobre la pista. No obstante, es importante destacar la importancia fundamental de la corporización en la generación del significado.

Para Masciotra et al. (2008) conocer es actuar. Por lo tanto, el conocimiento es significativo ya que es un componente de la acción que se desarrolla mediante un proceso dinámico y activo, "un conocimiento-acción que es el corazón de la relación cuerpo-mente" (p. 144). La inteligencia enactiva se desarrolla en situación, en el hacer y es "adaptativa, emocional, racional, cognitiva, distribuida, evolutiva y multiforme" (p. 85). En palabras de los autores "el pianista ejecuta en actos, piensa en actos: piensa con sus manos, las posibilidades que ellas emprenden" (p. 53).

Para facilitar la compresión al lector de lo que es el conocimiento enactivo, tomaremos algunas frases de Bardy y Mottet (2006), es así como ellos indican lo siguiente:

El conocimiento enactivo es información adquirida a través de las interacciones que se producen entre las conexiones percepción-acción y el ambiente (...) El conocimiento enactivo es adquirido a través de movimientos intuitivos de los cuales a menudo no somos conscientes. El conocimiento enactivo es intrínsecamente multimodal porque las acciones motrices modifican los estímulos del conjunto de sistemas perceptivos (...) El conocimiento enactivo no es simbólico ni icónico y es directo en el sentido que es natural e intuitivo. Está basado en la experiencia y en las consecuencias perceptivas de las acciones motrices (p. 9).

La experiencia enactiva corresponde a una expresión de la mente en el lenguaje corporal que se refleja en gestos y movimientos que en la mayoría de los casos son inconscientes durante la ejecución. El conocimiento enactivo del deportista no solo son las sensaciones que percibe y adquiere en su interacción con el entorno en el cual actúa, ellas son la base de la gestación de significados.

Para Warren (2006a) el conocimiento enactivo esta relacionado con el término presencia perceptiva que significa percibir la información invariante para detectar directamente las intenciones y posibilidades que nos brindan los objetos en relación con la dinámica de una actividad determinada. El conocimiento enactivo se expresa de una manera tácita o silenciosa en el experto durante el desarrollo de la acción.

A la hora de responder, la gran mayoría de las respuestas motrices y decisiones de los deportistas, emergen de manera óptima y acertada. Ellos poseen un conocimiento declarativo y, sobre todo, procedimental sobre cómo proceder, pero son incapaces de explicar sus actuaciones, todo se reduce a sus sensaciones. Es destacable la expresión de los deportistas 
cuando mencionan que han tenido buenas sensaciones en el agua, al golpear la pelota, en un salto determinado, sobre la bicicleta o al danzar al compás de la música. Según Todaro, Faste, Portillo-Rodriguez y Bergamasco (2007) el darse cuenta de esas sensaciones antes y durante la acción es lo que contribuiría a una optimización del aprendizaje y de sus rendimientos.

Uno de los modelos menos conocido en las Ciencias del Deporte y que presenta un gran interés es el que Dreyfus y Dreyfus, 1986) presentaron sobre el desarrollo de la pericia (The Skill Model), y cuyo ámbito de aplicación va dirigido a todos los contextos en los que se busca alcanzar el máximo nivel de pericia, como es el caso del deporte. Para estos autores el progreso individual atraviesa una serie de 5 niveles denominados: Principiante, Principiante avanzado, Competente, Eficiente y Experto. La superación de estos niveles es la consecuencia de la historia de acoplamientos entre el agente y el mundo. Las disposiciones para actuar son corporeizadas logrando que el deportista se fusione con su medio en rendimientos sobresalientes.

Para Dreyfus (2002) durante el proceso de adquisición de habilidades, se van incorporando gradualmente reglas en el accionar del cuerpo a medida que se incrementa la pericia. No obstante, el Experto se desprende de éstas reglas funcionando de un modo inteligente y sin representaciones ya que lo que verdaderamente se almacena son tendencias para responder. El experto no solo comprende la situación instantáneamente, sino que también ve de forma inmediata e intuitiva cómo responder (Ruiz, 2012). Sus disposiciones corporeizadas se ponen al servicio de la situación, son la expresión enactiva de la cognición corporeizada.

\section{La enacción como programa de investigación}

El progreso de un programa de investigación vinculado con la enacción debe seguir algunos fundamentos epistemológicos. Se mencionan solo algunas ideas recogidas del planteamiento de Varela (2000) que se presentan a continuación:

- La información no existe de antemano en un mundo predefinido $\mathrm{y}$, por consiguiente, la representación no ejerce un rol fundamental. Como consecuencia, los estudios y experiencias están enfocadas a analizar el presente vivo, en donde el individuo y el mundo se especifican de manera recíproca y se transforman constantemente.

- El individuo enactúa con el mundo y en conjunto, hacen emerger un significado que es producto de una historia viable de acoplamientos estructurales del sistema mismo.

- La cognición creativa se desarrolla en un mundo natural donde la ambigüedad y la incertidumbre están presentes. Por tanto, el ser humano requiere el permanente uso del sentido común contextual para adquirir y moldear un conocimiento que evoluciona constantemente.

- El mundo y el perceptor son dependientes e inseparables, lo que puede observarse en los ejemplos de experiencias de sustitución sensorial, como cuando una per- sona invidente debe aprender a ver activamente a través de vibraciones o del tacto. En ellas la percepción es acción perceptualmente guiada.

- Con respecto a la emergencia de los estados cognitivos, el autor menciona: "la percepción y la acción están encarnadas en los procesos sensorimotores (que son autoorganizantes), por lo tanto, es natural postular que las estructuras cognitivas surgen de esquemas recurrentes de actividad sensorimotriz" (pp. 233-234).

En continuidad con las ideas precedentes, Stewart, Gapenne y Di Paolo (2010) explicitan las características más relevantes que identifican y diferencian al enactivismo de otros enfoques corporeizados. Su identidad como paradigma se ve reflejada en las siguientes directrices:

- La enacción busca comprender "la articulación entre corporeización de nivel básico y la cognición humana de alto nivel" (p. viii).

- La enacción busca conectar la subjetividad de la experiencia vivida en primera persona con los datos objetivos obtenidos en tercera persona. La reflexividad es un problema contradictorio ya que existe una gran diferencia entre lo que percibe el organismo durante su propia experiencia y lo que percibe un observador externo de esa misma experiencia.

- La enacción "proporciona un marco conceptual extenso articulando varios dominios y niveles de organización que están implicados en la ciencia cognitiva” (p. xiv). Por ejemplo, niveles micro y macro, autopoiesis y cognición social, metabolismo y mente.

- La enacción aspira a "una circulación hermenéutica entre diversos enfoques, en la cual cada uno conserva su autonomía y su validez" (p. xv).

\section{Realidad virtual e interfaces}

Una de las vertientes de investigación que ha tenido un gran desarrollo en los últimos años consiste en crear interfaces de realidad virtual que permiten registrar y estudiar como evoluciona el comportamiento que vivencia un sujeto al experimentar y practicar una actividad.

Recientemente Gravel (2006) ha comentado las implicaciones de la enacción dentro de la comunidad científica, destacando en este interés la red europea Enactive Interfaces. Este proyecto reunió a numerosos laboratorios dedicados a investigar en torno a la noción de enacción, a la relación hombremáquina y a las consecuencias perceptivas o visuales del movimiento. El diseño y uso de estos sistemas de interfaces enactivas permitirían una considerable aportación al aprendizaje de las incitaciones para la acción- affordances (Stoffregen, Bardy y Mantel, 2006).

También contribuirían a que el pensamiento kinestésico pueda devenir más consciente gracias al entrenamiento que utiliza este tipo de tecnología (Faste, Todaro y Bergamasco, 2008). El proyecto SKILLS FP6 ha continuado en la línea 
del proyecto precedente y se caracteriza por estudiar el aprendizaje y la interdependencia entre las habilidades sensorio-motrices y las habilidades cognitivas (Bardy et al., 2010).

El uso y desarrollo de interfaces enactivas favorecería en un futuro cercano el incremento de la percepción quinestésica de los deportistas. Es muy probable que ellos aun no sean conscientes de la dimensión de lo que realmente hacen y que de momento sólo es accesible a los ojos del investigador cuando visiona y analiza las imágenes grabadas a alta frecuencia (e.g., $300 \mathrm{~Hz}$ cada $3.33 \mathrm{~ms}$ ).

La creación de tecnología enactiva podría proporcionar un feed-back durante la acción para que el atleta sea capaz de darse cuenta de lo que hace con su cuerpo y con la integración de sus modalidades sensoriales. Por ejemplo en el caso de un tenista, ser capaz de sentir la textura del puño de la raqueta, la solidez de la pista al tocarla con los pies, el sonido del golpeo contrario y de ver cómo la pelota comienza a volar en el inicio de su salto preparatorio antes de su desplazamiento.

En este sentido, Todaro et al. (2007) proponen la utilización de simulaciones que se proyecten en tiempo real para que el deportista pueda comparar sus movimientos actuales con los movimientos óptimos o deseados. Un posible diseño podría implicar un feed-back visual, táctil-vibratorio o acústico. De esta manera, el tenista podría sentir si sus movimientos son ejecutados en el timing justo o si manifiestan pequeños desfases temporales durante la realización de los ajustes de sus pies o en la regulación de la cabeza de la raqueta en su golpeo. Es importante también dejar claro que el análisis del tenista no debe quedar exclusivamente en un análisis de la dimensión sensorio-motora. Ya que él responde como un todo armónico donde es imposible separar su cuerpo de su mente o vice-versa. Durante un partido en un punto muy disputado, el jugador manifiesta un tono emocional acorde con la situación y el marcador. Realiza movimientos adoptando una postura específica. Ve y oye al mismo tiempo que posee planes, metas, una interioridad y una intencionalidad.

\section{Conclusión}

La gran variedad de acciones, fenómenos y dominios presentes en las actividades físicas y deportivas, hace muy difícil la unificación teórica que permita ver un puente de unión entre percepción, acción y cognición. Cada enfoque posee una pertinencia mayor frente a un tipo de fenómenos y de

\section{Referencias}

Abernethy, B. (1998). Perception-action relationships and the development of motor expertise. Science et Motricité, 28, 6-8.

Abernethy, B. y Sparrow, W. A. (1992). The rise and fall of dominant paradigms in motor behaviour research. En J. J. Summers (Ed.), Approaches to the study of motor control and learning (pp. 3-45). North Holland: Elsevier Science.

Araújo, D. (2013). The study of decision-making behavior in sport. Revista Internacional de Ciencias del Deporte-RICYDE, 31, 1-4. doi:10.5232/ricyde2013.031 tareas. Enfoques postcognitivos como la teoría ecológica ha demostrado experimentalmente una gran eficacia al explicar la regulación perceptivo-motriz en las acciones rápidas y de intercepción. La teoría de los sistemas dinámicos asociada con el modelo de manipulación de restricciones proporciona una explicación a la emergencia, a la adquisición y al desarrollo de la coordinación de los movimientos complejos. Sin embargo, su abandono de las referencias a la cognición hace difícil su aceptación como un enfoque que contemple a la persona en su totalidad.

Será interesante seguir indagando las técnicas orientales en las que se da énfasis a que el practicante sienta sus movimientos, relaje su cuerpo, no piense en lo que tiene que realizar sino que simplemente actúe, como es el caso del Tai Chi Chuan o de tantas otras artes marciales, samatha o el cultivo de la mente y las técnicas de meditación (Wallace, 2002). Sin duda recogen muchas de las ideas que los partidarios de la enacción defienden y que pueden dar pistas para el estudio del pensamiento kinestésico, el papel de la conciencia y la intuición en la acción.

La polémica existente entre los científicos del deporte, los psicólogos y los filósofos sobre el papel de la cognición, ha generado todo un movimiento postcognitivo que propone asumir que la cognición está anclada y enraizada en la dinámica sensomotriz de las interacciones entre el deportista, en su cuerpo y en su medio, es decir que enactúa en y con el mundo en el que vive, lo que se nos presenta como una opción interpretativa interesante de considerar (Stewart et al., 2010).

Junto con otros enfoques que Rowland (2010) recoge en su libro, el enactivismo propone en la actualidad un enfoque alternativo que permitiría un acercamiento a una mejor comprensión global del deportista. Éste ya no sería analizado solamente como un complejo perceptivo-cognitivo-motor, sino como una persona que mediante su accionar modifica o construye el mundo (su deporte) para solventar las contingencias y problemas que en éste se presentan (Penelaud, 2010).

Es posible que éste enfoque permita un progreso en el campo del comportamiento motor y deportivo, y en la comprensión de cómo actúan, evolucionan, aprenden y se desempeñan los deportistas en sus diferentes etapas de formación hasta alcanzar la pericia. El debate está abierto y su traducción en experiencias y estudios que apoyen o modifiquen estos postulados están todavía pendientes.

Araújo D., Davids K. y Hristovski R. (2006). The ecological dynamics of decision making in sport. Psychology of Sport and Exercise, 7, 653-676. doi:10.1016/j.psychsport.2006.07.002

Avilés, C., Ruiz, L. M. y Benguigui, N. (2006) ¿Qué conocemos sobre el comportamiento anticipatorio de los jugadores de tenis expertos durante el resto de un primer servicio? En D. Cabello, A. Lees, G. Torres e I. Roldán (Eds.), Colección Congresos n ${ }^{\circ}$ 2: IV World Congress of Science and Racket Sports (pp. 1-10). Madrid: Alto Rendimiento. 
Barandiaran, X., Di Paolo, E. y Rohde, M. (2009): Defining agency: Individuality, normativity, asymmetry, and spatio-temporality in action. Adaptive Behavior, 17, 367-386.

Bardy, B. G. (2006). Perception et régulation du mouvement humain: Plaidoyer pour une biologie physique. Journal de la Société de Biologie, 200, 135-143.

Bardy, B. G. (2011). Learning new skills in multimodal enactive environments. En B. G. Bardy, J. Lagarde y D. Mottet (Eds.), Proceedings of the 2011 International SKILLS Conference (pp. 1-4). Paris: EDP Sciences. doi.org/10.1051/bioconf/20110100008

Bardy, B. G., Delignières, D., Lagarde, J., Mottet, D. y Zelic, G. (Julio, 2010). An enactive approach to perception-action and skill acquisition in virtual reality environments. Trabajo presentado en la Third International Conference on Applied Human Factors and Ergonomics. Miami, FL, USA.

Bardy, B. G. y Mottet, D. (Noviembre, 2006). Introduction. Presentada en la Third International Conference on Enactive Interfaces: Enaction \& Complexity. Montpellier.

Beek, P. J., Jacobs, D., Daffertshofer, A. y Huys, R. (2003). Expert performance in sport: Views on expert performance in sport from the joint perspectives of ecological psychology and dynamical systems theory. En J. L. Starkes y K. A. Ericsson (Eds.), Expert performance in sports: Advances in research on sport expertise (pp. 321-344). Champaign, IL: Human Kinetics.

Benguigui, N. (1997). Effet de la pratique d'un sport de balle sur le développement des processus perceptifs impliqués dans les actions d'interception (Tesis doctoral inédita). Faculté des sciences du sport, Université de Poitiers, France.

Berthoz, A. (1997). Le Sens du Mouvement. Paris: Odile Jacob.

Bootsma, R. J. (Abril, 1993). L'approche écologique de la perception et l'action. Actes des Journées Spécialisées de l'ACAPS. Neurosciences comportementales des APS "Approches cognitives et écologiques", Poitiers.

Bootsma R. J. (1994). Le couplage Perception-Action dans les activités d'interception. Trabajo inédito. Habilitation à diriger des Recherches. Université de la Méditerranée. Marseille, Francia.

Bootsma, R. J., Houbiers, M., Whiting, H. T. A. y Van Wieringen, P. C. W. (1991). Acquiring an attacking forehand drive: The effects of static and dynamic environmental conditions. Research Quarterly for Exercise and Sport, 62, 276-284.

Bootsma, R. J. y Van Wieringen, P. C. W. (1990). Timing an attacking forehand drive in table tennis. Journal of Experimental Psychology: Human Perception and Performance, 16, 21-29.

Bruner, J. S. (1980). Investigaciones sobre el desarrollo cognitivo (Trad. A. Maldonado). Madrid: Pablo del Río. (Trabajo original publicado en 1966).

Carlton, L. G. (1992). Visual processing time and the control of movement. En L. Proteau y D. Elliot (Eds.), Vision and motor control (pp. 3-31). Amsterdam: Elsevier Science.

Chow, J. Y., Davids, K., Hritoski, R., Araújo, D. y Passos, P. (2010). Nonlinear pedagogy: Learning design for self-organizing neurobiological systems. New Ideas in Psychology, 29, 189-200. doi: 10.1016/j.newideapsych.2010.10.001

Colley, A. (1989). Learning motor skills: Integrative cognition and action. En A. Colley y J. Beech (Eds.), Acquisition and Performance of Cognitive Skills (pp. 167-189). Chichester: John Wiley y Sons.

Crognier, L. y Féry, A. (2007). 40 ans de recherches sur l'anticipation en tennis: Une revue critique. Science et Motricité, 62, 9-36.

Davids, K., Button, C. y Bennett, S. (2008). Dynamics of skill acquisition: A constraints-led approach. Champaign, IL: Human Kinetics.

Davids, K., Handford, C. y Williams, M. (1994). The natural physical alternative to cognitive theories of motor behavior: An invitation for interdisciplinary research in sports science? Journal of sports Sciences, 12, 495-528.

Davids, K., Williams, A.M., Button, C. y Court, M. (2001). An integrative modeling approach to the study of intentional movement behaviour. En R. N. Singer, H. Hausenblas y C. Janelle (Eds.), Handbook of sport psychology (pp. 144-173). New York: John Wiley and Sons.

Dewey, J. (1929). Experience and nature. (2 Ed.). New York: Dover

De Jaegher, H., y Di Paolo, E. (2007). Participatory sense-making: An enactive approach to social cognition. Phenomenology and the Cognitive Sciences, 6, 485-507.

Di Paolo, E., Rohde, M. y De Jaegher, H. (2010). Horizons for the enactive mind: Values, social interaction, and play. En J. Stewart, O. Gapenne y
E. Di Paolo (Eds.), Enaction: Towards a New Paradigm for Cognitive Science (pp. 33-87). Cambridge, MA: MIT Press.

Dreyfus, H. (2002) Intelligence without representation - Merleau-Ponty's critique of mental representation. The relevance of phenomenology to scientific explanation. Phenomenology and the Cognitive Sciences, 1, 367-383.

Dreyfus, H. y Dreyfus, S. (1986). Mind over machine: The power of human intuition and expertise in the era of the computer. New York: The Free Press.

Farrow, D. y Abernethy, B. (2003). Do expertise and the degree of perception-action coupling affect natural anticipatory performance? Perception, 32, 1127-1139.

Faste, H., Todaro, E. y Bergamasco, M. (Noviembre, 2008). Kinesthetic thinking: Heightening enactive experience. Trabajo presentado en la Fifth International Conference on Enactive Interfaces, Pisa.

García, A. y Calvo, F. (2011). Introducción. La cognición extendida en contexto. En A. Clark y D. Chalmers. La mente extendida (Trad. A. García y F. Calvo) (pp. 7-57). Oviedo: KRK Ediciones. (Trabajo original publicado en 2002).

Gravel, P. (5 de Junio de 2006). Concept d'« enaction»: La connaissance du monde passé par l'action. Le Devoir, p. A4.

Gibson, J. J. (1986). The ecological approach to visual perception. Hillsdale, N. J: Lawrence Erlbaum Associates, Inc. (Trabajo original publicado en 1979).

Handford, C., Davids, K., Bennett, S. y Button, C. (1997). Skill acquisition in sport: Some applications of an evolving practice ecology. Journal of Sports Sciences, 15, 621-640.

Havelange V., Lenay C. y Stewart J. (2003). Les représentations: Mémoire externe et objets techniques. Intellectica, 35, 115-131.

Hutto, D. D. (2013). Radically enactive cognition in our grasp. En Z. Radman (Ed.), The hand: An organ of the mind (pp. 227-252). Cambridge, MA: MIT Press.

Ibáñez-Gijón, J., Travieso, D. y Jacobs, D. M. (2011). El enfoque ecológico como marco conceptual y metodológico para el diseño de programas de entrenamiento deportivo. Revista de Psicología del Deporte, 20, 667-688.

Jacobs, D. M. y Michaels, C. F. (2007). Direct learning. Ecological Psychology, 19, 321-349

Keil, D., Holmes, P., Bennett, S., Davids, K. y Smith, N. (2000). Theorie and pratique in sport psychology and motor behavior needs to be constrained by integrative modelling of brain behavior. Journal of Sports Sciences, 18, 433-443

Laurent, E. y Ripoll, H. (2009). Extending the rather unnoticed Gibsonian view that 'perception is cognitive': Development of the enactive approach to perceptual-cognitive expertise. En D. Araújo, H. Ripoll y M. Raab (Eds.), Perspectives on Cognition and Action in Sport (pp. 133-146). Hauppauge, New York: Nova Publishers.

Lee, D. y Reddish, P. E. (1981). Plummeting gannets: A paradigm of ecological optics. Nature, 293, 293-294.

Marr, D. (1985). La visión. Madrid: Alianza. (Trabajo original publicado en 1982).

Masciotra, D., Roth, W.-M. y Morel, D. (2008). Énaction: Apprendre et enseigner en situation (Trad. Groupe De Boeck). Bruxelles: De Boeck. (Trabajo original publicado en 2007).

Maturana, H. y Varela, F. (1996). El árbol del conocimiento. Las bases biológicas del conocimiento humano. Madrid: Debate.

Maturana, H. y Varela, F. (2004). De máquinas y seres vivos. Autopoiesis: La organización de lo vivo. Buenos Aires: Editorial Universitaria y Editorial Lumen.

Merleau-Ponty, M. (1985). Fenomenología de la Percepción. Barcelona: PlanetaAgostini. (Trabajo original publicado en 1945).

Michaels, C. F. y Beek, P. (1995). The state of ecological psychology. Ecologi cal Psychology, 7, 259-278.

Michaels, C. F. y Carello C. (1981). Direct Perception. Englewood Cliffs, N. J.: Prentice-Hall.

Milner, A. D. y Goodale, M. A. (1995). The visual brain in action. Oxford, England UK: Oxford University Press.

Moe, V. F. (2005). A philosophical critique of classical cognitivism in sport: From information processing to bodily background knowledge. Journal of the Philosophy of Sport, 32, 155-183.

Montagne, G. (2005). Prospective control in sport. International Journal of Sport Psychology, 36, 127-150. 
Montagne, G., Bastin, J. y Jacobs, D. M. (2008). What is visual anticipation, and how much does it rely on the dorsal stream? International Journal of Sport Psychology, 39, 149-156.

Moreno, F. J. y Ordoño, E. M. (2009). Aprendizaje motor y síndrome general de adaptación. Motricidad. European Journal of Human Movement, 22, 1-21.

Newell, K. M. (1985). Coordination control and skill. En D. Goodman, R. B. Wilberg y I. M. Franks (Eds.), Differing perspectives on motor learning, memory and control (pp. 295-317). Amsterdam: Elsevier Science.

Newell, K. M. (1986). Constraints on the development of coordination. En M. G. Wade y H. T. A. Whiting (Eds.), Motor Development in children: Aspects of coordination and control (pp. 341-360). Dordrecht, Netherlands: Martinus Nijhoff.

Nistch, J. R. y Munzert, J. (2002). Aspectos del entrenamiento de la técnica desde la perspectiva de la teoría de la acción: Aproximaciones a un modelo integrador. En J. R. Nitsch, A. Neumaier, H. Mareales y J. Mester (Eds.), Entrenamiento de la técnica (pp. 129-191). Barcelona: Paidotribo.

Noë, A. (2004). Action in perception. Cambridge: The MIT Press.

Noë, A. (2010). Fuera de la cabeza: Por qué no somos el cerebro: Y otras lecciones de la biología de la consciencia (Trad. N. d'Amonville). Barcelona: Kairós (Trabajo original publicado en 2009).

Norman, J. (2002). Two visual systems and two theories of perception: An attempt to reconcile the constructivist and ecological approaches. Behavioral and Brain Sciences, 25, 73-144.

O'Regan, J. K. y Noë, A. (2001). A sensorimotor account of vision and visual consciousness. Behavioral and Brain Sciences, 24, 939-1031.

Oullier, O., Lagarde, J. F., Jantzen, K. J. y Kelso, J. A. S. (2006). Coordination dynamics: (in)stability and metastability in the behavioural and neural systems. Journal de la Société de Biologie, 200, 145-167.

Paillard, J. (1991). The cognitive penetrability of sensorimotor mechanisms: A key problem in sport research. International Journal of Sport Psychology, 22, 244250.

Palut, Y., Zanone, P. G. y Gurdjos, P. (2004). Modes de déplacement spontané en tennis. Science \& Motricité, 51, 69-83.

Pasquinelli, E. (2007). Enactive knowledge. En A. Luciani y C. Cadoz (Eds). Enaction and enactive interfaces, a bandbook of terms (pp. 93-95). Grenoble: Enactive Systems Books.

Penelaud, O. (2010). Le paradigme de l'énaction aujourd'hui: Apports et limites d'une théorie cognitive " révolutionnaire ». Revue Transdisciplinaire de Plasticité Humaine, 18, 1-38. Recuperado de http://plasticites-sciencesarts.org/PLASTIR/Penelaud\%20P18.pdf

Peña. A. (2010). The Dreyfus model of clinical problem-solving skills acquisition: A critical perspective. Medical Education Online, 15, 1-11. doi: 10.3402/meo.v15i0.4846

Pérez, D. (2010). Los límites de las tesis de la mente extendida: Agencia, autonomía y autoridad epistémica. Revista de Filosofía Factótum, 7, 62-75. Recuperado de http://www.revistafactotum.com/revista/f_7/articulos LFactotum_7_6_David_Perez.pdf

Poincairé, H. (1907). La science et l'hyphotesis. Paris: Flammarion.

Ripoll, H. (2009). What is the impact of knowledge on player's behaviour? En D. Araújo, H. Ripoll y M. Raab (Eds.), Perspectives on Cognition and Action in Sport (pp. 89-93). New York: Nova Science.

Récopé, M. (2006). Normativité et sensibilité: une perspective généalogique d'étude du mouvement et de l'action. Motricité et schèmes des volleyeurs mobilisés en défense. Trabajo inédito, Habilité à Diriger des Recherches en STAPS, Université Blaise Pascal, Clermont-Ferrand, Francia.

Récopé, M., Fache, H., y Rix, G. (2008). Norme propre et exercice corporel: le cas d'un volleyeur. Revue Corps, 4, 105-110.

Rowland, M. (2010). The New Science of the Mind. From extended mind to embodied phenomenology. Cambridge: MIT Press

Ruiz, L. M. (2003a). Competencia motriz, dinamismo y complejidad en educación física: Parte I. Retos: Nuevas tendencias en Educación Física, Deporte y Recreación, 5, 39-43.

Ruiz, L. M. (2003b). Competencia motriz, dinamismo y complejidad en educación física: Parte II. Retos: Nuevas tendencias en Educación Física, Deporte y Recreación, 6, 5-12.

Ruiz, L. M. (2012). Si quieres decidir bien, no pienses, actúa. El papel de los procesos intuitivos en el deporte, Gymnasium. Revista Lusófona de Educaçao Física, Desporto e Saude, 3, 118-138.

Ruiz, L. M., Sánchez, M., Durán, J. y Jiménez, C. (2006). Los expertos en el deporte: Su estudio y análisis desde una perspectiva psicológica. Anales de psicología, 22, 132-142.
Savelsbergh, G. J. P., Van der Kamp, J., Oudejans, R. R. D. y Scott, M. A. (2004). Perceptual learning is mastering perceptual degrees of freedom. En A. M. Williams y N. J. Hodges (Eds.), Skill Acquisition in Sport: Research Theory and Practice (pp. 374-389). London: Taylor \& Francis.

Schmidt, R. A. (1991). Motor learning and performance: From principles to practice. Champaign, IL: Human kinetics.

Stewart, J., Gapenne, O. y Di Paolo, E. (2010). Enaction: Towards a new paradigm for cognitive science. Cambridge, MA: The MIT Press.

Stoffregen, T. A., Bardy, B. G. y Mantel, B. (2006). Affordances in the design of enactive systems. Virtual Reality, 10, 4-10.

Summers, J. J. (2004). A historical perspective on skill acquisition. En A. M. Williams y N. J. Hodges (Eds.), Skill acquisition in sport: Research, theory and practice (pp. 1-26). London: Routledge.

Temprado, J. J. (1994). Méthodes de chronométrie mentale et traitement de l'information. Science et Motricite, 22, 23-33.

Temprado, J. J. y Laurent, M. (1995). Approches cognitive et écologique de l'apprentissage des habilités motrices en sport. En H. Ripoll, J. Bilard, M. Durand, J. Keller, M. Levêque y P. Therme. (Eds.), Psychologie du sport: Questions actuelles. (pp. 223-236). Paris: Revue EPS.

Temprado, J. J. y Montagne, G. (2001). Les coordinations perceptivo-motrices. Paris: Armand Colin.

Tenenbaum, G. y Land, W.M. (2009). Mental representations as an underlying mechanism of human performance. En M. Raab, J.G. Johnson y H.R. Heekeren (Eds.), Progress in brain research: Mind and Motion-the bidirectional link between thought and action (pp. 251-266). Amsterdam: Elsevier.

Thompson, E. (2011). Living Ways of Sense-Making [Suplemento]. Pbilosopby Today SPEP, 114-123.

Todaro, E., Faste, H., Portillo-Rodriguez, O. y Bergamasco, M. (Octubre, 2007). The hand dance: $A$ didactic performance platform. Trabajo presentado en la International Cultural Heritage Informatics Meeting, Toronto.

Torrents, C. y Balagué, N. (2007). Repercusiones de la teoría de los sistemas dinámicos en el estudio de la motricidad humana. Apunts. Educació Física $i$ Esports, 87, 7-13.

Varela, F. (1984). Living ways of sense-making: A middle path for neuroscience. En P. Livingston (Ed.), Order and Disorder: Proceedings of the Stanford International Symposium (pp. 208-224). Stanford: Anma Libri.

Varela F. (1996). Invitation aux sciences cognitives (Trad. P. Lavoie) Paris: Éditions du Seuil. (Trabajo original publicado en 1988).

Varela, F. (2000). El fenómeno de la vida. Santiago: Dolmen.

Varela, F., Thompson, E. y Rosch, E. (2005). De cuerpo presente: Las ciencias cognitivas y la experiencia bumana (Trad. C. Gardini) ( $3^{\mathrm{a}}$ reimp.). Barcelona: Gedisa. (Trabajo original publicado en 1991).

Vickers, J. (2009). Advances in coupling perception and action: The quiet eye as a bidirectional link between gaze, attention, and action. Progress in Brain Research, 174, 279-288.

Wallace, B.A. (2002). The Buddhist tradition of Samatha: Methods for refining and examining consciousness. En F. Varela y J. Shear (Eds.), The view from within. First-person approaches to the study of consciousness (175-187). Thorverton: Imprint Academic.

Warren, W. H. (1984). Perceiving affordances: Visual guidance of stair climbing. Journal of experimental psychology: Human perception and performance, 10, 683-703

Warren, W. H. (Noviembre, 2006a). Enactive Knowledge: Sensory-motor expectancies or perception-action invariants? Trabajo presentado en la Third International Conference on Enactive Interfaces: Enaction \& Complexity, Montpellier.

Warren, W. H. (2006b). The dynamics of perception and action. Psychological Review, 113, 358-389.

Wheeler, M. (2001). Two threats to representation. Synthese, 129, 211-231.

Whiting, H. T. A. (1984). Human motor actions: Bernstein reassessed. Amsterdam: North Holland.

Williams, A. M. y Davids, K. (1998). Perceptual expertise in sport: Research, theory and practice. En H. Steinberg, I. Cockerill, y A. Dewey (Eds.), What sport psychologist do (pp. 48-57). Leicester, UK: British Psychological Society.

Williams, A. M., Davids, K. y Williams, J. G. (1999). Visual perception and action in sport. London: E. \& F. N. Spon.

Wilson, M. (2002). Six views of embodied cognition. Psychonomic Bulletin \& Review, 9, 625-636.

Withagen, R. y Chemero, A. (2009). Naturalizing perception: Developing the Gibsonian approach to perception along evolutionary lines. Theory \& Psycho$\log$, 19, 363-389.

(Artículo recibido: 21-9-2012; revisado: 25-10-2012; aceptado: 25-02-2013) 